\title{
HETARYL DISPLACEMENT IN 3,6-DISUBSTITUTED 1,2,4,5-TETRAZINES WITH ANHYDRO BASES OF $N$-METHYLQUINALDINIUMS
}

\author{
Gennady L. Rusinov*, Rashida I. Ishmetova, Ilya N. Ganebnykh, Oleg N. Chupakhin \\ Institute of Organic Synthesis of RAS, 22, S. Kovalevskoy St., \\ Ekaterinburg, 620219, Russian Federation, Fax: +7 (343) 37411 89, E-mail: rusinov@ios.uran.ru
}

\begin{abstract}
Bis(4-R-3,5-dimethylpyrazol-1-yl)-s-tetrazine, 3,6-bis(imidazol-1-yl)-s-tetrazine, 3,6bis(benzotriazol-1-yl)-s-tetrazine,3,6-bis(4-methylimidazol-l-yl)-s-tetrazine react with anhydro bases of 1-methylquinaldium and 1,6-dimethylquinaldium to form products of $C$-nucleophilic substitution of azolyl fragment or Carboni-Lindsey reaction followed by subsequent pyridine ring cleavage in the quinoline moiety.
\end{abstract}

\section{Introduction}

Previously it was reported that $s$-tetrazines, namely 3,6-diphenyl- and 3,6-di-2-pyridyl-1,2,4,5tetrazines, being highly reactive dienes with inverse electron demand, can be used as traps for exomethylene anhydro bases formed in situ from azinium cations. ${ }^{1,2}$ At the same time we have found that 3,6-bis-(4-R-3,5-dimethylpyrazol-1-yl)-1,2,4,5-tetrazines participate in [4+2]cycloaddition reaction with enamines in acetonitrile to give [4+2]cycloaddition products - a variety of dihydro and aromatic pyridazines. ${ }^{3}$ On the other hand, it has been known that enamine may also react as $C$ nucleophile in nucleophilic substitutions. But there are few data on nucleophilic substitution in tetrazines with $C$-nucleophiles. Some examples of such substitution can be found in papers. ${ }^{4-8}$ While widely used solvents for $\mathrm{S}_{\mathrm{N}}{ }^{\text {ipso }}$-reactions are polar methanol, ethanol or acetonitrile, cycloaddition better proceeds in non-polar solvents like toluene.

\section{Discussions}

The purpose of the present work was to investigate the reactions of 3,6-bis(4-R-3,5-dimethylpyrazol1-yl)-s-tetrazines 1a-c and easily obtained from them 3,6-bis(benzotriazol-1-yl)-, 3,6-bis(imidazol-1yl)-, and 3,6-bis(4-methylimidazol-yl)-s-tetrazines $2,3,{ }^{10} 4$ with anhydro bases of 1 methylquinaldinium and 1,6-dimethylquinaldinium. The tetrazines 1-3 are promising compounds for synthesis of a variety of novel $s$-tetrazine derivatives due to their ability to participate in nucleophilic substitutions as was already shown for $\mathrm{N}$ - and $\mathrm{O}$-nucleophiles.

The anhydro bases mentioned above were prepared in situ from corresponding iodides in acetonitrile by action of equimolar amount of triethylamine. It has been found that $C$-nucleophilic substitution of one of two dimethylpyrazolyl, or benzotriazolyl, or imidazolyl moieties easily proceeds in acetonitrile (Scheme-1) at room temperature with good or even excellent yield (50-98\%).

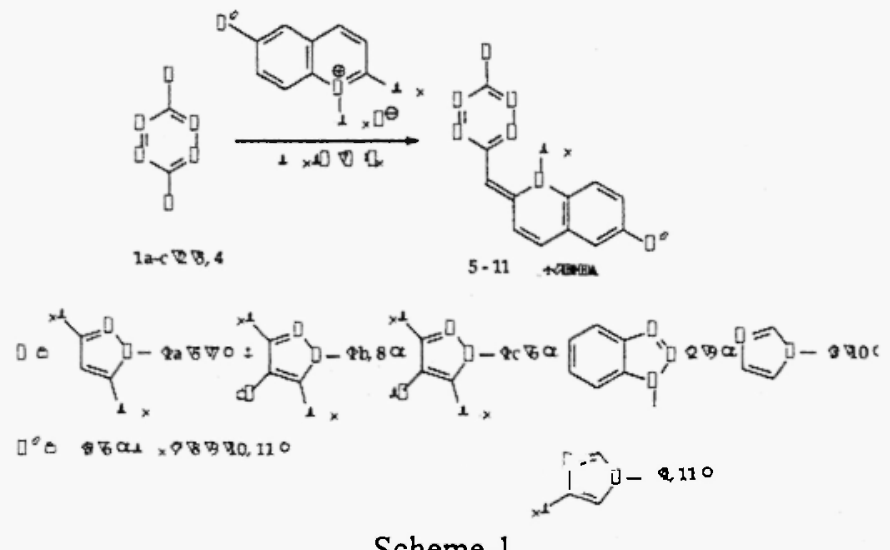

Scheme-1 
In case of the most active tetrazine 4 the substitution completely finished after 10-15 minutes, while other tetrazine derivatives reacted for 1-3 $\mathrm{h}$ at room temperature. It indicates that 4-methylimidazol-1yl moiety in tetrazines is more preferable for substitution than 3,5-dimethylpyrazol-1-yl ones.

The structure of the obtained dark-brown crystalline compounds 5-11 was determined by ${ }^{1} \mathrm{H}$ NMR spectroscopy. In ${ }^{1}$ NMR spectra of all substitution products 5-11 we observed signals of methylidene protons as singlets at $\delta 5.85-5.95 \mathrm{ppm}$. Protons at $\mathrm{C} 3$ of dihydroquinolinium ring resonated as well resolved doublets at $\delta 8.74-8.78 \mathrm{ppm}$ while protons at $\mathrm{C} 4$ and other aromatics gave multiplets.

Thus all tetrazines 1-4 in acetonitrile smoothly gave substitution products.

We wanted to find out if substitution reaction will compete with cycloaddition in less polar solvents than acetonitrile, or not. So we studied the reaction of 3,6-bis-(4-R-3,5-dimethylpyrazol-1-yl)-1,2,4,5tetrazine (1a) with 1,6-dimethylquinaldinium iodide in toluene on heating. At least three reaction products were detected by TLC (acetonitrile:benzene 1:1; Silufol (®). We failed to isolate and identify one of them $\left(R_{f} 0.18\right)$ formed in small quantities. The cycloaddition product 12 was obtained in yield $20 \%$, while the major product was tetrazine 7 (yield $68 \%$ ).

So, the reaction of the tetrazine $1 \mathrm{a}$ with 1,6-dimethylquinaldinium iodide in toluene proceeds according to Scheme-2:

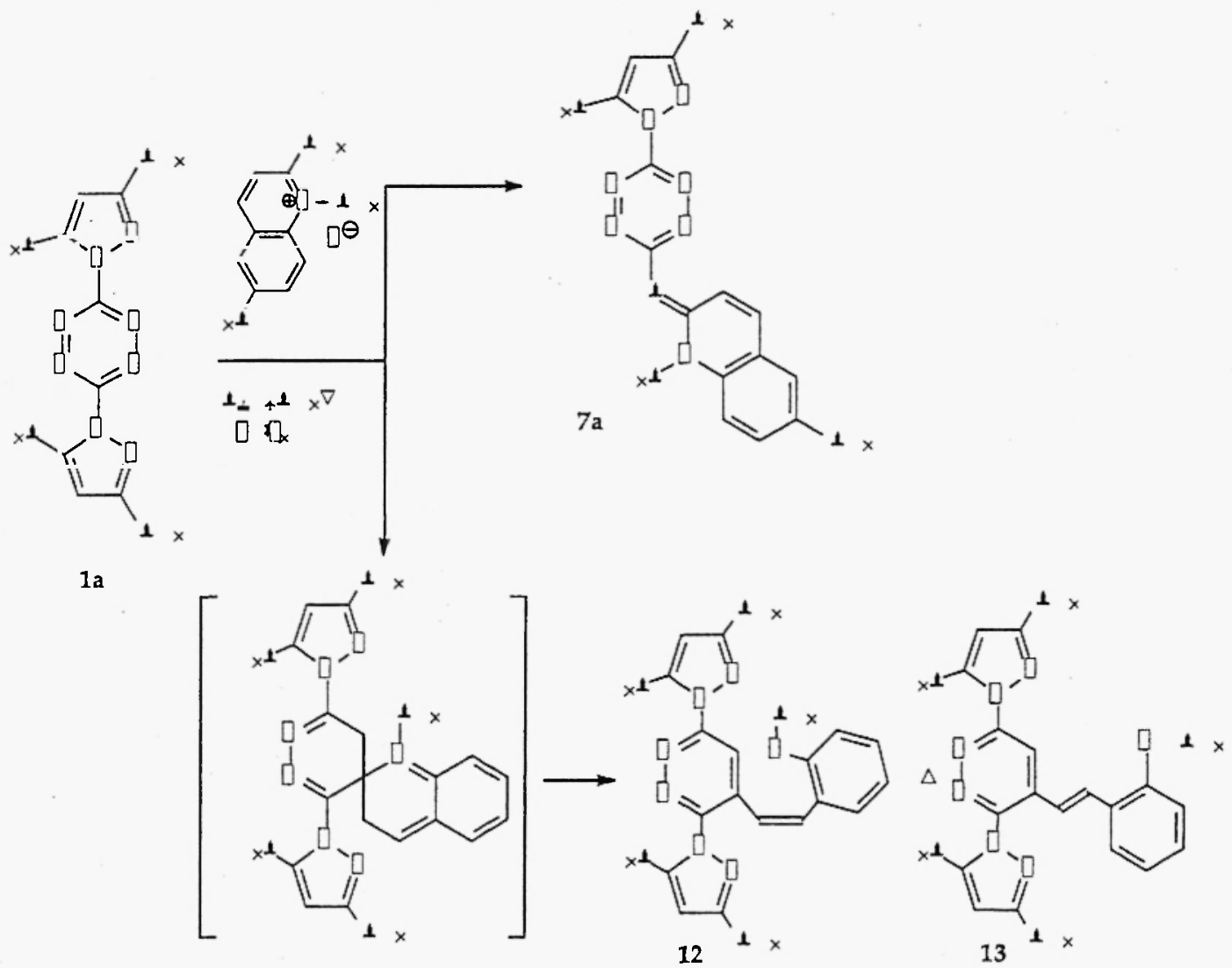

Scheme-2

A route to pyridazine 12 involves two stages similar to the previously reported for $3,6-$ diphenyl-s-tetrazine ${ }^{2}$ : spiro-compound formation and subsequent pyridine ring cleavage. Therefore, initially cis-isomer should form. We have recorded the ${ }^{1} \mathrm{H}$ NMR spectrum of the crude product obtained after its extraction from the reaction mixture by hexane. The spectrum had two sets of 
signals in a ratio approximately $10: 2$ according to integration curves. Olefinic protons of major isomer resonated as doublets at $\delta 6.41$ and $6.77 \mathrm{ppm}(J=11.9 \mathrm{~Hz})$, respectively. This indicates that cis-form (12) dominates in the mixture of trans- and cis-isomers. Similar doublets of trans-form (13) were downfield shifted $(6.83$ and $7.34 \mathrm{ppm} ; J=16.1 \mathrm{~Hz}$ ). Hydrogen atom at pyridazine ring in 12 resonated also as a doublet at $8.01 \mathrm{ppm}$ having long-range constant $J=0.6 \mathrm{~Hz}$ with methylidene proton.

In the similar reaction of tetrazine 4 under the same conditions, in toluene, we have isolated only the product of $C$-nucleophilic substitution - tetrazine 11 (yield 79\%), although the TLC also showed the formation of small amounts of corresponding pyridazine derivative.

All of the novel compounds (5-11) have sharp melting points, are water insoluble, sparingly soluble in alcohols, readily soluble in chloroform. The obtained products have satisfactory $\mathrm{CHN}$ analysis data and are TLC-pure.

\section{Experimental}

${ }^{\mathrm{I}} \mathrm{H}$ NMR spectra were measured on a Bruker DRX-400 (400 MHz) spectrometer in $\mathrm{CDCl}_{3}$. All signals are expressed in ppm $(\delta)$ with TMS as an internal standard. Melting points (uncorrected) were obtained on a Boetius apparatus.

3,6-Bis(4-R-3,5-dimethylpyrazol-1-yl)-1,2,4,5-tetrazines (1a-c) were prepared according to the known methods. ${ }^{311}$ 3,6-Bis(benzotriazol-1-yl)- and 3,6-bis(imidazol-1-yl)-1,2,4,5-tetrazines $(2,3$ ) were obtained as described in the literature. ${ }^{10}$

3,6-Bis(4-methylimidazol-1-yl)-s-tetrazine (4).

To a suspension of $2.0 \mathrm{mmol}(0.54 \mathrm{~g})$ of tetrazine $1 \mathrm{a}$ in $10 \mathrm{ml}$ of dry $\mathrm{CH}_{3} \mathrm{CN} 6 \mathrm{mmol}(0.49 \mathrm{~g})$ of 4methylimidazole was added. After 3-5 min. of stirring fine yellow crystals precipitated from the solution, which were filtered off, washed by $\mathrm{CH}_{3} \mathrm{CN}$ and dried to yield $0.27 \mathrm{~g}(56 \%)$ of pure 4 .

General procedure for preparing dihydroquinolines $(4,5,6,7,8,9,10,11)$.

$1 \mathrm{Mmol}$ of corresponding tetrazine 1a-c, 2,3 was suspended in $15 \mathrm{ml}$ of acetonitrile. Then $1 \mathrm{mmol}$ of 1,6-dimethylquinaldinium iodide or 1-methylquinaldinium iodide and $0.2 \mathrm{ml}$ of triethylamine were added. The reaction mixture color changed from intensive red to dark brown. The reaction was stirred for $3 \mathrm{hrs}$. Solvent was evaporated. The residue was triturated with distilled water, filtered off and recrystallized from DMF. Yield: 52-98\%. Physical data are listed below.

(2\{2-[3,6-Bis-(3,5-dimethylpyrazol-1-yl)-pyridazin-4-yl)-ethyl\}-4-methylphenyl)-methylamine (12).

A mixture of 3,6-bis(3,5-dimethylpyrazol-1-yl)-1,2,4,5-tetrazine $1 \mathrm{a}(0.270 \mathrm{~g}, 1.4 \mathrm{mmol})$ and of 1,6-dimethylquinaldinium iodide $(0.312 \mathrm{~g}, 1.0 \mathrm{mmol})$ were suspended in $20 \mathrm{ml}$ of toluene. After adding of $0.2 \mathrm{ml}$ of triethylamine the formed brown mixture was refluxed for $4.5 \mathrm{~h}$. Then the toluene was removed. The brown residue was triturated and washed by water, filtered off and dried. Then the title compound was extracted by $40 \mathrm{ml}$ of hexane at $20^{\circ} \mathrm{C}$. Hexane was removed from filtrate, and the residue was triturated by acetonitrile to yield $0.08 \mathrm{~g}$ of pure cis-compound $12(19 \%)$. Physical data of are listed below. Undissolved brown residue after extraction was identical to tetrazine 7 (yield 68\%).

4: yield: $56 \%$ m.p. $208-209^{\circ} \mathrm{C} .{ }^{1} \mathrm{H}$ NMR: 2.37 (s, $6 \mathrm{H}, 2 \mathrm{CH}_{3}$ ), $7.71,8.66$ (both d, $2 * 1 \mathrm{H}, \mathrm{CH}^{\text {imidazole }}$ ). Found (\%): C, 49.52; H, 4.00; N, 46.46. Calc. for $\mathrm{C}_{10} \mathrm{H}_{10} \mathrm{~N}_{8}(\%)$ : C, 49.58; H, 4.16; N, 46.26.

5: yield: $98 \%, \mathrm{mp} 223-224^{\circ} \mathrm{C}$. ${ }^{1} \mathrm{H}$ NMR: $2.36,2.62$ (both s, $2 * 3 \mathrm{H}, 2 \mathrm{CH}_{3}{ }^{\mathrm{P}}$ ); $3.67\left(\mathrm{~s}, 3 \mathrm{H}, \mathrm{NCH}_{3}\right), 5.89$ (s, $1 \mathrm{H}, \mathrm{CH}=), 6.09\left(\mathrm{~s}, 1 \mathrm{H}, \mathrm{CH}^{4-\mathrm{Pz}}\right), 7.04-7.54\left(\mathrm{~m}, 5 \mathrm{H}, \mathrm{CH}^{\text {quinoline }}\right), 8.78\left(\mathrm{~d}, 1 \mathrm{H}, \mathrm{CH}^{\mathrm{C} 3 \text {-Quinoline }}, J=9.8\right.$ $\mathrm{Hz}$ ). Found (\%): C, 65.20; H, 5.26; N, 29.65. Calc. for $\mathrm{C}_{18} \mathrm{H}_{17} \mathrm{~N}_{7}(\%): \mathrm{C}, 65.23 ; \mathrm{H}, 5.17 ; \mathrm{N}, 29.60$. 
6: yield: $55 \%$, mp $257-258^{\circ} \mathrm{C} .{ }^{1} \mathrm{H}$ NMR: $2.38,2.65$ (both s, $2 * 3 \mathrm{H}, 2 \mathrm{CH}_{3}{ }^{\mathrm{Pz}}$ ), $3.73\left(\mathrm{~s}, 3 \mathrm{H}, \mathrm{NCH}_{3}\right.$ ), 5.94 (s, $1 \mathrm{H}, \mathrm{CH}=), 7.16-7.45\left(\mathrm{~m}, 5 \mathrm{H}, \mathrm{CH}^{\text {quinoline }}\right), 8.78\left(\mathrm{~d}, 1 \mathrm{H}, \mathrm{CH}^{\text {C3-Qunoline }}, J=9.8 \mathrm{~Hz}\right)$. Found $(\%)$ : $\mathrm{C}$, 59.05; H, 4.42; N, 26.73. Calc. for $\mathrm{C}_{18} \mathrm{H}_{16} \mathrm{ClN}_{7}(\%)$ : C, 59.09; $\mathrm{H}, 4.41 ; \mathrm{N}, 26.81$.

7: yield: $74 \%$, mp $205-208^{\circ} \mathrm{C}$. ${ }^{1} \mathrm{H}$ NMR: $2.37,2.38,2.64$ (all s, $3 * 3 \mathrm{H}, 2 \mathrm{CH}_{3}{ }^{\mathrm{P}}+\mathrm{CH}_{3}{ }^{\mathrm{C} 6-\text { Quinoline }}$ ), 3.06 (s, $\left.3 \mathrm{H}, \mathrm{NCH}_{3}\right), 5.88(\mathrm{~s}, 1 \mathrm{H}, \mathrm{CH}=), 6.10\left(\mathrm{~s}, 1 \mathrm{H}, \mathrm{CH}^{4-\mathrm{Pz}}\right), 7.22-7.31\left(\mathrm{~m}, 4 \mathrm{H}, \mathrm{CH}^{\text {quinoline }}\right), 8.75\left(\mathrm{~d}, 1 \mathrm{H}, \mathrm{CH}^{\mathrm{C3}-}\right.$ Quinoline $J=9.8 \mathrm{~Hz}$ ). Found (\%): $\mathrm{C}, 65.47 ; \mathrm{H}, 5.53 ; \mathrm{N}, 27.98$. Calc. for $\mathrm{C}_{19} \mathrm{H}_{19} \mathrm{~N}_{7}(\%): \mathrm{C}, 66.06 ; \mathrm{H}$, $5.54 ; \mathrm{N}, 28.39$.

8: yield: $52 \%$, mp $228-231^{\circ} \mathrm{C} .{ }^{1} \mathrm{H}$ NMR: $2.38,2.39,2.63$ (all s, $3 * 3 \mathrm{H}, 2 \mathrm{CH}_{3}{ }^{\mathrm{Pz}}+\mathrm{CH}_{3}{ }^{\mathrm{C} 6-\mathrm{Q} \text { unoline }}$ ), 3.71 (s, $\left.3 \mathrm{H}, \mathrm{NCH}_{3}\right), 5.89(\mathrm{~s}, 1 \mathrm{H}, \mathrm{CH}=), 7.22-7.35\left(\mathrm{~m}, 4 \mathrm{H}, \mathrm{CH}^{\text {quinoline }}\right), 8.75\left(\mathrm{~d}, 1 \mathrm{H}, \mathrm{CH}^{\mathrm{C} 3-\text { Qunoline }}, J=9.8 \mathrm{~Hz}\right)$. Found (\%): C, 53.47; H, 4.13; N, 22.89. Calc. for $\mathrm{C}_{19} \mathrm{H}_{18} \mathrm{BrN}_{7}(\%)$ : C, 53.78; H, 4.28; N, 23.11.

9: yield: $70 \%$, mp $242-245^{\circ} \mathrm{C}$. ${ }^{1} \mathrm{H}$ NMR: $2.40\left(\mathrm{~s}, 3 \mathrm{H}, \mathrm{CH}_{3}{ }^{\mathrm{Cb} \text {-quinoline }}\right), 3.73\left(\mathrm{~s}, 3 \mathrm{H}, \mathrm{NCH}_{3}\right), 5.89(\mathrm{~s}, 1 \mathrm{H}$, $\mathrm{CH}=)$, 7.20-7.38 (m, 6H, 4CH $\left.\mathrm{CH}^{\text {Quinoline }}+2 \mathrm{H}^{\text {benzotriazole }}\right), 7.94\left(\mathrm{~m}, 1 \mathrm{H}, \mathrm{CH}^{\text {benzotriazole }}\right), 8.64(\mathrm{~m}, 1 \mathrm{H}$, $\left.\mathrm{CH}^{\text {benzotriazole }}\right), 8.72\left(\mathrm{~d}, 1 \mathrm{H}, \mathrm{CH}^{\text {C3-Quinoline }}, J=9.8 \mathrm{~Hz}\right.$ ). Found (\%): $\mathrm{C}, 65.26 ; \mathrm{H}, 4.73 ; \mathrm{N}, 30.36$. Calc. for $\mathrm{C}_{20} \mathrm{H}_{16} \mathrm{~N}_{8}(\%): \mathrm{C}, 65.20 ; \mathrm{H}, 4.38 ; \mathrm{N}, 30.42$.

10: yield: $81 \%$, mp $239-240^{\circ} \mathrm{C} .{ }^{1} \mathrm{H}$ NMR: $2.40\left(\mathrm{~s}, 3 \mathrm{H}, \mathrm{CH}_{3}{ }^{\mathrm{C}-\text {-Quinoline }}\right), 3.73\left(\mathrm{~s}, 3 \mathrm{H}, \mathrm{NCH}_{3}\right), 5.88(\mathrm{~s}, 1 \mathrm{H}$, $\mathrm{CH}=), 7.28-7.37\left(\mathrm{~m}, 5 \mathrm{H}, 4 \mathrm{CH}^{\text {Quinoline }}+1 \mathrm{H}^{\text {imidazole }}\right), 7.94\left(\mathrm{t}, 1 \mathrm{H}, \mathrm{CH}^{\text {imidazole }}, J=1.3 \mathrm{~Hz}\right), 8.64(\mathrm{t}, 1 \mathrm{H}$, $\left.\mathrm{CH}^{\text {imddazole }}, J=1.3 \mathrm{~Hz}\right), 8.72\left(\mathrm{~d}, 1 \mathrm{H}, \mathrm{CH}^{\mathrm{C} 3 \text {-Quinoline }}, J=9.8 \mathrm{~Hz}\right.$ ). Found (\%): $\mathrm{C}, 64.34 ; \mathrm{H}, 4.77 ; \mathrm{N}, 30.69$. Calc. for $\mathrm{C}_{17} \mathrm{H}_{15} \mathrm{~N}_{7}(\%)$ : C, 64.34; $\mathrm{H}, 4.76 ; \mathrm{N}, 30.90$.

11: yield: $98 \%$; m.p. $248-250^{\circ} \mathrm{C} .{ }^{1} \mathrm{H}$ NMR: 2.36 (d, $\left.3 \mathrm{H}, \mathrm{CH}_{3}{ }^{\text {inidazole }}, J=0.9 \mathrm{~Hz}\right), 2.40$ (s, $3 \mathrm{H}$, $\left.\mathrm{CH}_{3}{ }^{\text {quinoline }}\right) ; 3.71\left(\mathrm{~s}, 3 \mathrm{H}, \mathrm{NCH}_{3}\right), 5.87(\mathrm{~s}, 1 \mathrm{H}, \mathrm{CH}=), 7.25-7.28\left(\mathrm{~m}, 2 \mathrm{H}, 2 \mathrm{CH}^{\text {quinoline }}\right), 7.34(\mathrm{t}, 2 \mathrm{H}$, $\left.2 \mathrm{CH}^{\text {quinoline }}\right), 7.63\left(\mathrm{t}, 1 \mathrm{H}, \mathrm{H}^{\mathrm{Cs} \text {-imidazole }}\right), 8.57\left(\mathrm{~d}, 1 \mathrm{H}, \mathrm{CH}^{\mathrm{C} 2 \text {-imidazole }}, J=1.1 \mathrm{~Hz}\right), 8.70\left(\mathrm{~d}, 1 \mathrm{H}, \mathrm{CH}^{\text {quinoline }}, J=\right.$ $9.8 \mathrm{~Hz}$ ), . Found (\%): $\mathrm{C}, 65.34 ; \mathrm{H}, 5.29 ; \mathrm{N}, 29.44$. Calc. for $\mathrm{C}_{18} \mathrm{H}_{17} \mathrm{~N}_{7}(\%): \mathrm{C}, 65.24 ; \mathrm{H}, 5.17 ; \mathrm{N}$, 29.59 .

12: (cis-form) yield: $19 \%$, mp $143^{\circ} \mathrm{C}$. ${ }^{1} \mathrm{H}$ NMR: $2.16,2.23,2.29,2.65$ (all s, $4 * 3 \mathrm{H}, 2 \mathrm{CH}_{3}{ }^{\mathrm{C} 3-\mathrm{Pz}}+$ $\mathrm{NHCH}_{3}+\mathrm{CH}_{3}{ }^{A x}$ ), 2.26, 2.59 (d, $2 * 3 \mathrm{H}, 2 \mathrm{CH}_{3}{ }^{\mathrm{Cs}-\mathrm{Pz}}, J=0.5 \mathrm{~Hz}$ ), $4.25-4.30$ (br.s, $1 \mathrm{H}, \mathrm{NHCH}_{3}$ ), 5.97, 5.99 (both s, $2^{*} \mathrm{CH}, 2 \mathrm{CH}^{4-\mathrm{Pz}}$ ), $6: 41,6.77$ (both d, $\left.2^{*} 1 \mathrm{H}, \mathrm{CH}=\mathrm{CH}-\mathrm{Ar}, J=11.9 \mathrm{~Hz}\right), 6.42\left(\mathrm{~d}, 1 \mathrm{H}, \mathrm{CH}^{\mathrm{C} 3} \cdot\right.$ Ar, $J=8.3 \mathrm{~Hz}$ ), 6.81 (br.s, $\left.1 \mathrm{H}, \mathrm{CH}^{\mathrm{Cb} \cdot \mathrm{As}}\right), 6.95$ (dd, $\left.1 \mathrm{H}, \mathrm{CH}^{\mathrm{CA} \cdot \mathrm{Ar} r}, J=8.3 ; 2.0 \mathrm{~Hz}\right), 8.01$ (d, $1 \mathrm{H}$, $\mathrm{CH}^{\text {pyridazine }}, J=0.6 \mathrm{~Hz}$ ). Found (\%): C, 69.51; H, 6.59; N, 23.65. Calc. for $\mathrm{C}_{24} \mathrm{H}_{27} \mathrm{~N}_{7}(\%): \mathrm{C}, 69.71 ; \mathrm{H}$, 6.85; N, 23.71. $\mathrm{R}_{\mathrm{f}} 0.5\left(\mathrm{CH}_{3} \mathrm{CN}\right.$ :benzene 1:1; Silufol $\left.\mathbb{8}\right)$.

13: ${ }^{\mathrm{H}} \mathrm{NMR}$ (from a mixture of 12 and 13 ): $2.24,2.35,2.35,2.87$ (all s, $4 * 3 \mathrm{H}, 2 \mathrm{CH}_{3}{ }^{\mathrm{C}-\mathrm{Pz}}+\mathrm{NHCH}_{3}+$ $\mathrm{CH}_{3}{ }^{\mathrm{As}}$ ), 2.32, 2.76 (d, $2 * 3 \mathrm{H}, 2 \mathrm{CH}_{3}{ }^{\mathrm{CS} \cdot \mathrm{Pz}}, J=0.6 \mathrm{~Hz}$ ), $4.25-4.30$ (br.s, $\mathrm{NHCH}_{3}$ ), 6.08, 6.09 (both $\mathrm{s}$, $\left.2 * \mathrm{CH}, 2 \mathrm{CH}^{4-\mathrm{Pz}}\right), 6.83,7.34$ (both d, $2 * 1 \mathrm{H}, \mathrm{CH}=\mathrm{CH}-\mathrm{Ar}, J=16.1 \mathrm{~Hz}$ ), $6.58\left(\mathrm{~d}, 1 \mathrm{H}, \mathrm{CH}^{\mathrm{C} 3-\mathrm{Ar}}, J=8.3\right.$ $\mathrm{Hz})$, 7.03-7.15 (m, $\left.2 \mathrm{H}, \mathrm{CH}^{\mathrm{C} 4, \mathrm{C} 6-\mathrm{Ar}}\right), 8.41\left(\mathrm{~d}, 1 \mathrm{H}, \mathrm{CH}^{\text {pyridazine }}, J=0.6 \mathrm{~Hz}\right)$.

\section{Acknowledgements}

This work was supported by the Russian Foundation for Basic Research (grant N 04-03-96011) and the International Science and Technology Center (Project No. 708).

\section{References}

1. E.G. Kovalev, I.Ya. Postovskii, G.L. Rusinov, I.L. Shegal. Khimia Geterotsikl. Soedin. 11, 1462 (1981)

2. E.G. Kovalev, G.L. Rusinov, V.A. Anufriev, L.G. Egorova. Chem. Heterocycl. Compd. (Engl. Transl.) 26, 1038 (1990)

3. G.L. Rusinov, R.I. Ishmetova, N.I. Latosh, I.N. Ganebnykh, O.N. Chupakhin, V.A. Potemkin. Rus. Chem. Bull. 49, $355(2000)$

4. A. Mangia, F. Bortesi, U. Amendola. J.Heterocycl.Chem., 14, 587 (1977)

5. C.M. Hudson, M.E. Neubert, A.M. Lackner, J.D. Margerum, E. Sherman. Liquid Crystals, 19, 871 (1995)

6. H. Yamanaka, S. Ohba. Heterocycles, 31, 895 (1990)

7. S.C. Benson, L. Lee, L. Yang, J.K. Snyder. Tetrahedron, 56, 1165 (2000).

8. Z. Novák, A. Kotschy. Org. Lett., 5, 3495 (2003)

9.. G.L. Rusinov, R.I. Ishmetova, I.N. Ganebnykh, O.N. Chupakhin, G.G. Aleksandrov, I.A. Litvinov, D.B. Krivolapov. Heterocycl. Commun. 9, 39 (2003)

10. G.L. Rusinov, N.I. Latosh, I.N. Ganebnykh, R.I. Ishmetova, N.K. Ignatenko, O.N. Chupakhin. Russ. J. Org. Chem, in press (2005)

11. M.D. Cobum, G.A. Buntain, B.W. Harris, M.A. Hiskey, K.-Y. Lee and D.G. Ott, J. Heterocycl. Chem. 28, 2049 (1991)

Received on September 15, 2005 